\title{
El reto de la seguridad humana en América Latina: El problema de la violencia endémica en la región ${ }^{1}$
}

\section{The challenge of human security in Latin America: The problem of endemic violence in the region}

\author{
Cristina Churruca Muguruza ${ }^{2}$ \\ Universidad de Deusto (España)
}

Recibido: 10-06-14

Aprobado: 25-06-14

\section{Resumen}

A partir de una revisión de los últimos informes, así como de las publicaciones más relevantes que han surgido en los últimos años, este trabajo aborda el fenómeno de la violencia en América Latina y sus consecuencias en términos de seguridad

\footnotetext{
${ }^{1}$ Este trabajo se ha realizado en el marco del proyecto de investigación "Un nuevo marco conceptual para la protección del desplazamiento forzoso de población" [Referencia CSO 201125202], financiado por el Programa Nacional de Proyectos de Investigación Fundamental, en el marco del VI Plan Nacional de Investigación Científica, Desarrollo e Innovación Tecnológica 2008-2011.

${ }^{2}$ (cristina.churruca@deusto.es) Profesora de Relaciones Internacionales e investigadora del Instituto de Derechos Humanos de la Universidad de Deusto. Es directora del Máster Universitario en Acción Internacional Humanitaria en la Universidad de Deusto y Coordinadora del consorcio de Universidades del máster NOHA en Acción Internacional Humanitaria. Ha sido profesora e investigadora en el Instituto del Derecho Internacional de la Paz y los Conflictos Armados en la Universidad de Bochum, Alemania. Sus áreas principales de investigación son la seguridad humana, la protección desde una perspectiva de derechos y la construcción de la paz. Sus publicaciones más recientes incluyen: CHURRUCA MUGURUZA, C., "EUFOR TCHAD/RCA mission on the protection of civilians: a distinctive EU way to peace operations", in Maria Grazia Gallantino and Raquel Freire (eds.), MANAGING CRISIS, MAKING PEACE. Towards a Strategic EU Vision for Security and Defence Rethinking Peace and Conflict Studies Series edited by Oliver P. Richmond, London, Routledge, 2014; CHURRUCA MUGURUZA, C., "Vulnerabilidad y protección en la acción humanitaria" en BARRANCO, M.C. y CHURRUCA, C. (ed.), Vulnerabilidad y protección de los derechos humanos, Valencia, Tirant lo Blanch, Valencia, 2014.pp.45-70; Cristina Churruca y Cristina de la Cruz, "La responsabilidad de las empresas en el desplazamiento forzado de población: ¿parte del problema o de la solución? Experiencias en Colombia" en Carmen Márquez Carrasco (ed.), España y la implementación de los Principios Rectores de las Naciones Unidas sobre empresas y derechos humanos: oportunidades y desafios / Spain and the Implementation of the United Nations Guiding Principles on Business and Human Rights: Challenges and Opportunities, editorial Huygens, Barcelona , 2014, pp. 631-650.
} 
humana. Con este fin se introduce en primer lugar el concepto de violencia, para mostrar un panorama de la misma en la región más allá de las tasas conocidas de homicidios y poner en evidencia su impacto en la vida de las personas. América Latina sufre una violencia endémica que tiene su campo privilegiado de acción en las zonas urbanas y, sobre todo, en aquellas pobres, segregadas y excluidas de las grandes ciudades, donde la presencia del Estado es o prácticamente inexistente o insuficiente e inoperante. Además de las consecuencias directas que tiene la violencia en la vida y los medios de vida de las personas, ésta produce un sentimiento de temor y vulnerabilidad, y repercute de forma negativa en el espacio público y el tejido social. Se señala en particular una consecuencia poco estudiada de la violencia y cuya magnitud todavía no está reconocida por los Estados de la región: el desplazamiento forzado de población por causa de grupos y estructuras criminales.

Palabras-clave: Seguridad humana, América Latina, violencia endémica, violencia urbana, desplazamiento forzado.

\begin{abstract}
From a review of recent reports as well as the most relevant publications that have emerged in recent years, this paper addresses the phenomenon of violence in Latin America and its consequences in terms of human security. To this end, it first introduces the concept of violence to show a panorama of violence in the region beyond the known homicide rates and evidence the impact of violence in the lives of people. Latin America suffers from endemic violence that has privileged the urban and, especially, the poor areas, segregated and excluded from the big cities where state presence is practically non-existent or inadequate and inoperative as its field of action. In addition to the direct consequences of violence on the lives and livelihoods of people, is produces a sense of fear and vulnerability and impacts negatively on the public space and the social fabric. A lesser known dimension of the violence which is underestimated by the States in the region is highlighted: the forced displacement of people due to criminal groups and structures.
\end{abstract}

Key-words: Human security, Latin America, endemic violence, urban violence, forced displacement. 


\section{Introducción}

La seguridad humana es una expresión que refleja la progresiva convergencia entre las agendas de paz y seguridad, desarrollo y derechos humanos. En los últimos años, se ha alcanzado un consenso internacional sobre el hecho de que la seguridad, los derechos humanos y el desarrollo son condiciones interdependientes e indispensables para la paz y el desarrollo sostenible y se refuerzan mutuamente. A pesar de importantes diferencias en intereses y perspectivas, todos los países miembros de las Naciones Unidas aprobaron incluir en el Documento Final de la Cumbre Mundial 2005 el doble objetivo de la seguridad humana, reconociendo la libertad frente a la necesidad, su concepción más amplia, y la libertad frente al temor, su concepción más restringida, como valores esenciales para las relaciones internacionales en el siglo XXI (NN.UU-AG 2005). La adopción de la resolución 66/290 de la Asamblea General el 10 de septiembre de 2012 ha supuesto otro paso importante en este consenso. El párrafo 3 de la resolución subraya no solo el "derecho de las personas a vivir en libertad y con dignidad, libres de la pobreza y la desesperación", sino "la responsabilidad primordial del Estado, de los gobiernos, de asegurar la supervivencia, los medios de subsistencia y la dignidad de sus ciudadanos".

La seguridad humana se refiere precisamente, según la definición propuesta por la Comisión de la Seguridad Humana en su informe Human Security Now, publicado en 2003, a la protección de esos tres elementos que conforman el núcleo vital de todas las vidas humanas: los elementos básicos de supervivencia, dignidad y medios de vida. La seguridad humana quiere decir que "todas las personas, en particular las vulnerables, tienen derecho a vivir libres del temor y la miseria, a disponer de iguales oportunidades para disfrutar de todos sus derechos y a desarrollar plenamente su potencial humano" (CSH 2003:7). Por ello la protección del núcleo vital de todas las vidas humanas de manera que mejoren las libertades humanas y la realización humana es una responsabilidad primordial del Estado. La seguridad humana de sus ciudadanos debe ser, por tanto, un objetivo fundamental de todos los gobiernos ${ }^{3}$. La función de la comunidad internacional consiste en completar la labor de los gobiernos y proporcionarles el apoyo necesario, cuando lo soliciten, a fin de fortalecer su capacidad para proteger a sus ciudadanos.

Los desafíos a la seguridad humana varían de región a región, de individuo a individuo y se manifiestan de diferentes maneras según las especificidades culturales, de género, generacionales, étnicas, etc. Por ello, no es posible

\footnotetext{
${ }^{3}$ Sobre el concepto de seguridad humana y la diferenciación entre su definición amplía y restringida véase Cristina Churruca, Human Security as a policy framework: Critics and Challenges [en Anuario de Accion Humanitaria y Derechos Humanos/Yearbook on Humanitarian Action and Human Rights 2007, Bilbao,Universidad de Deusto, 2007], págs. 15-35.
} 
hablar de la seguridad humana en América Latina en sentido general, pues ésta depende del país, de la región, del grupo poblacional específico. No obstante, sí se pueden identificar algunos desafíos críticos y generalizados del contexto latinoamericano que afectan los derechos de las personas y ponen en riesgo su posibilidad de una vida digna, libre del miedo y de la miseria. Informes recientes sobre el desarrollo de América Latina, en donde se toman en cuenta los retos y las oportunidades que la región tiene por delante, coinciden en señalar que los dos grandes retos de la región tienen que ver con la desigualdad y con la violencia (CEPAL 2013; PNUD 2012). Aunque se ha producido un crecimiento económico y una estabilidad financiera notables, así como se ha avanzado en la reducción de la pobreza, la desigualdad y el desempleo, la violencia se ha mantenido en unos niveles muy altos e incluso ha aumentado en los últimos años. Las encuestas de victimización y percepción llevadas a cabo por el Proyecto de Opinión Pública de América Latina (LAPOP) y el PNUD ponen de manifiesto que los ciudadanos de la región señalan el delito y la violencia como factores que limitan sus oportunidades reales y su derecho a vivir libres de temor y de amenazas (LAPOP-PNUD 2012).

América Latina es efectivamente la región donde se presentan los más altos niveles de violencia del mundo. En su conjunto la región sufre unas tasas de violencia mucho más altas que otras regiones, con más de 100.000 homicidios registrados por año según el estudio global sobre homicidio, Global Study on Homicide 2013 de la Oficina de Naciones Unidas sobre Drogas y Crimen, UNODC en sus siglas en inglés (UNODC 2014). Se estima que desde los años 80 la mayoría de los países de la región tienen tasas de homicidio con niveles de epidemia según la clasificación de la Organización Mundial de la Salud. Pero durante los últimos años el nivel y la intensidad de la violencia ha aumentado en ciudades de Colombia y Brasil y en el Triángulo Norte de Centroamérica (TNCA), que abarca Guatemala, Honduras y El Salvador y México por la rápida expansión de grupos armados del crimen organizado, que incluye redes de narcotráfico, pandillas callejeras, maras y grupos criminales, que operan desde el nivel local hasta el transnacional. Las consecuencias humanitarias de este fenómeno son comparables a las experimentadas durante las guerras civiles que asolaron la región a mediados y finales de los 80 (ECHO 2013). La preocupación por la dimensión y el alcance de la violencia ha llevado al PNUD a dedicar su último Informe Regional de Desarrollo Humano para América Latina 2013-14 al desafío de garantizar la seguridad ciudadana. El informe Seguridad Ciudadana con Rostro Humano: Diagnóstico y propuestas para América Latina analiza y pone en evidencia el problema de la violencia en la región y ofrece recomendaciones para mejorar las políticas públicas ${ }^{4}$.

\footnotetext{
${ }^{4}$ La seguridad ciudadana se entiende en América Latina como una modalidad específica de la seguridad humana, relacionada con la seguridad personal y, más específicamente, con amenazas como 
La violencia tiene un gran impacto en la seguridad humana y en el desarrollo humano. Los Objetivos de Desarrollo del Milenio no prestaron la debida relación a los problemas de la violencia y a la complejidad de sus relaciones con la gobernabilidad, el estado de derecho y los derechos humanos. América Latina sufre una violencia de carácter endémico que afecta la vida de las personas y a su relación con las autoridades públicas. En un momento en que la comunidad internacional está comprometida en la definición de la Agenda de Desarrollo Post-2015 urge reconocer la verdadera dimensión del problema para poder abordarlo con las políticas adecuadas. Salvo para los estudiosos de la seguridad y los conflictos en América Latina el problema de la violencia en la región aparece eclipsado por las cifras de crecimiento económico y las buenas perspectivas de desarrollo. Empero, este tipo de violencia crónica y, como veremos, fundamentalmente urbana no se circunscribe sólo a América Latina, sino que afecta a cada vez más regiones del mundo, por lo que puede convertirse en una cuestión que afecta a la paz y seguridad internacionales. En este contexto el objetivo de este trabajo es contribuir a la comprensión del fenómeno de la violencia en América Latina y a sus consecuencias en términos de seguridad humana a partir de una revisión de los informes citados, así como de las publicaciones más relevantes que sobre este tema han surgido en los últimos años. Con este fin se introduce en primer lugar el concepto de violencia, para abordar posteriormente las características e impacto de la violencia en la vida de las personas.

\section{Una aproximación al concepto de violencia.}

La violencia está tan presente en nuestras vidas que se la percibe a menudo como un componente inevitable de la condición humana. No hay país ni comunidad a salvo de la violencia. La primera recapitulación general sobre el problema de la violencia a nivel mundial realizado por la OMS en el año 2002 reconoce que es "un azote ubicuo que desgarra el tejido comunitario y amenaza la vida, la salud y la felicidad de todos" (OMS 2002:11).

La complejidad del fenómeno de la violencia hace difícil formular un concepto preciso de lo que debe entenderse por ella. Por ello la literatura relativa a la violencia en muchas ocasiones elude la definición del término. Los trabajos relativos a la violencia en América Latina, por ejemplo, utilizan denominaciones que aluden a diversos tipos de violencia, como violencia delincuencial, violencia de gangs, violencia policial, violencia narcotraficante, narcoviolencia, violencia homicida y violencia excepcional entre otros, sin describir siempre el fenómeno o hacerlo de una forma comprensiva.

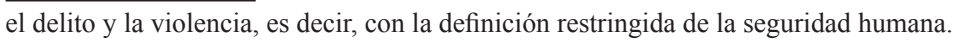


Las diferentes acepciones del término en el diccionario de la Real Academia Española tampoco la definen directamente, sino que se remiten a la "cualidad de violento", a la "acción y efecto de violentar o violentarse" o "acción violenta o contra el natural modo de proceder". La violencia presenta además una peculiaridad añadida a las dificultades para definirla: la de que es sobre todo una relación. Además de depender de diversas variables y circunstancias es una cuestión de apreciación. La noción de lo que son comportamientos aceptables e inaceptables, o de lo que constituye un daño, está influida por la cultura y sometida a una continua revisión a medida que los valores y las normas sociales evolucionan. Por otra parte, la violencia puede definirse de muchas maneras, según el tipo de violencia, quién la haga y con qué propósito. La clasificación utilizada en el Informe mundial sobre la violencia y la salud por ejemplo divide la violencia en tres grandes categorías según el autor del acto violento: violencia dirigida contra uno mismo, violencia interpersonal y violencia colectiva. Esta última es la infligida por grupos más grandes, como los Estados, grupos políticos organizados, milicias u organizaciones terroristas (OMS 2002:11).

Un problema añadido para entender el fenómeno de la violencia en toda su complejidad es la falta de indicadores adecuados. La mayoría de los estudios no cubren de forma comprensiva los distintos tipos de violencia. En general se centran en las muertes violentas relacionadas con los conflictos armados o con las muertes intencionadas. Los índices de homicidio son los indicadores más comunes y visibles ya que resultan los más completos, comparables y precisos para medir la violencia. La violencia conyugal o de pareja, mucho más frecuente y desafortunadamente más aceptable socialmente en muchos lugares, resulta más difícil de cuantificar. Sin embargo, las estimaciones de la OMS revelan que la violencia de pareja es el tipo más común de violencia contra la mujer, ya que afecta al $30 \%$ de las mujeres en todo el mundo, y en particular a algunas regiones entre las que se encuentran los países de América Latina (WHO 2013).

Además existen diferentes definiciones de la violencia. Julio Aróstegui distingue entre varios tipos de definiciones con el objeto de establecer sus límites (Aróstegui 1994: 25-29). Las definiciones restringidas u observacionales son aquellas que ciñen el contenido de la violencia a la acción directa normalmente mediante el uso de fuerza física, y que acarrea la producción de daño personal o material. Esta posición puede identificarse con la idea común, usual y popular, de la violencia. La crítica de este uso restrictivo de la violencia es que tiende a identificar «violencia» con «fuerza física». En este caso, la definición asocia determinantemente con la violencia la existencia de daño físico, es decir, es una definición «observacional», que atiende a los resultados visibles de la acción y no a su origen, propósito o efecto. El problema es que estas definiciones a veces 
llevan a excusar e incluso justificar ciertos actos de violencia de las personas privadas y a condenar como inmorales los actos de violencia ejercidos desde instancias públicas. Estas son las posiciones legitimistas o estrictas, que ponen el énfasis en la violencia como el uso de la fuerza que no tiene una legitimación o sanción legal. Sería violencia, pues, según esta visión, el uso de la fuerza de forma ilegítima o ilegal. La crítica a estas definiciones es que asocia este concepto al de delito. Aunque el delito y la violencia constituyen amenazas a la vida y a la integridad física y patrimonial de las personas deben entenderse como categorías analíticas distintas, dado que no todo delito es violento ni todo acto de violencia es tipificado como delito en las leyes. Asimismo, la violencia como categoría de análisis permite subrayar claramente un conjunto de motivaciones que suelen ser excluidas cuando se piensa en la categoría de delito asociado comúnmente a móviles de tipo económico.

Las definiciones amplias o expansivas tienen como modelo, siguiendo a Galtung, la llamada violencia estructural, entendida como la opresión y el sufrimiento social causados por la pobreza, la represión y la alienación generadas por el conjunto de estructuras que no permiten la satisfacción de las necesidades y se concreta, precisamente, en la negación de las mismas (Galtung 1969). Estas definiciones consideran que la violencia abarca un amplio espectro de injusticias y desigualdades sociales. La violencia es, por tanto, casi equiparable a toda situación de dominación. El problema de entender la violencia de una forma tan extensa es que no permite delimitar unas fronteras precisas de esta realidad.

Partiendo de estas consideraciones, a efectos de delimitar este complejo fenómeno, se propone la definición adoptada por la OMS que entiende por violencia:

"El uso deliberado de la fuerza física o el poder, ya sea en grado de amenaza o efectivo, contra uno mismo, otra persona o un grupo o comunidad, que cause o tenga muchas probabilidades de causar lesiones, muerte, daños psicológicos, trastornos del desarrollo o privaciones" (OMS 2002:5).

Esta definición a pesar de ser restringida es lo suficientemente amplia como para abarcar una amplia gama de actos que van más allá del acto físico para incluir las amenazas e intimidaciones y cubrir las distintas manifestaciones de la misma reconociendo que la clave de la violencia está en la violación de un derecho básico de la persona. Además, esta definición de violencia es muy similar a la que de forma más breve ha sido utilizada por los investigadores de América Latina (Briceño 2008: 3).

Así y todo, esta definición no es suficiente, como veremos más adelante, para comprender el alcance de la violencia en América Latina: su carácter recurrente, su implantación en diversos espacios sociales, sus potenciales 
efectos trans-generacionales, y el hecho de que destruye relaciones sociales y provoca comportamientos sociales perversos que tienden a naturalizarse entre grupos vulnerables. Tani Marilena sugiere el concepto de "violencia crónica" de Pearce para aproximarse a la complejidad del fenómeno de la violencia en la región (Pearce 2007:7). La autora considera como violencia crónica aquella que ocurre en contextos en que los niveles de violencia se miden en las dimensiones de intensidad, espacio y tiempo (Marilena 2013: 13).

Este tipo de violencia tiene un carácter endémico en la medida que se ha convertido en algo habitual en ciertos países y áreas de América Latina. Pero el problema de la violencia endémica y prolongada no se circunscribe sólo a América Latina, sino que se presenta en varios países de ingreso bajo y medio en el África sub-Sahariana, Asia y el Medio Oriente (Pearce y McGee 2011). Un creciente grupo de especialistas humanitarios y de desarrollo son conscientes de cómo algunas ciudades (Ciudad Juárez, Medellín, Río de Janeiro, Karachi y Tegucigalpa entre otras) sufren una violencia con graves consecuencias para la seguridad humana. Aunque no necesariamente afectados por los conflictos armados, estos y otros centros urbanos están experimentando niveles de violencia, a menudo en barrios y barriadas densamente pobladas, a la par de otros devastados por la guerra como Abidjan, Benghazi, Damasco o Mogadiscio. Las situaciones de violencia prolongada, conocidas también por el nombre de "Otras Situaciones de Violencia" (Other Situations of Violence, OSV), tienen graves implicaciones desde un punto de vista humanitario, porque generan altas cifras de muertos, heridos, población desplazada e incluso refugiada, con lo que comparten gran parte de las características de los conflictos armados convencionales (Muggha 2012; Savage y Muggah 2012; Hasow 2013).

\section{América Latina: una región caracterizada por la violencia homicida.}

América Latina ha experimentado históricamente niveles elevados de violencia. Desde mediados de la década de 1950 las tasas de homicidio han sido de cinco a ocho veces mayores que las de Europa y Asia como resultado de la violencia política. Pero a partir de la década de los 80 aumenta notablemente el número de homicidios en América Latina tanto en países que ya tenías altas tasas de homicidios, Colombia o El Salvador, como en aquellos con bajos índices como Costa Rica y Argentina. La magnitud que alcanzó el fenómeno en las décadas siguientes llevo a considerar este fenómeno como un problema de salud pública comparando la tasa de mortalidad por homicidio de algunos países con niveles epidémicos. Esta violencia que irrumpe en los años setenta y ochenta tiene un carácter distinto. Es una violencia calificada de social, por 
expresar conflictos sociales y económicos; pero no de política, pues no tiene una vocación de poder.

La OMS realizó el primer informe sobre el panorama de la situación de la violencia en las distintas regiones y países del mundo en 2002. Según la OMS, en el año 2000 1,6 millones de personas perdieron la vida en todo el mundo por actos violentos, lo que representa una tasa de casi el 28,8 por 100000 . Aproximadamente la mitad de estos fallecimientos se debieron a suicidios, casi una tercera parte a homicidios (casi medio millón) y aproximadamente una quinta parte a conflictos armados. Este informe reveló que las muertes violentas varían en función de los ingresos de los países. En los de ingresos bajos y medios eran más de dos veces superiores (32,1 por 100 000) a las de los países de altos ingresos (14,4 por 100 000). También las tasas mundiales ocultaban grandes variaciones entre las regiones de la OMS en África y en las Américas, las tasas de homicidio eran casi tres veces superiores a las de suicidio. Sin embargo, en Asia Sudoriental y en Europa, las tasas de suicidio eran más de dos veces superiores a las de homicidio, y en la del Pacífico Occidental casi sextuplican a las de homicidio. Mientras que en África las muertes derivaban en una proporción altísima de los conflictos armados la situación existente en América Latina era muy distinta. Los homicidios cometidos por la delincuencia organizada y las pandillas eran muy elevados en zonas de América Central y del Sur. Las tasas mundiales escondían también amplias variaciones dentro de los propios países: entre las poblaciones urbana y rural, entre las comunidades ricas y las pobres, y entre los diversos grupos raciales y étnicos. El homicidio se concentraba de forma alarmante en América Latina y las otras regiones entre la población joven, en particular en los varones (OMS 2002:16).

Diez años más tarde el Global Study on Homicide 2013 de la Oficina de Naciones Unidas sobre Drogas y Crimen ofrece un panorama no muy diferente. El número de personas que ha perdido la vida a causa de homicidios en todo el mundo permanece estable, casi medio millón de personas en 2012 y se mantienen las variaciones regionales. Más de una tercera parte de los homicidios (36\%) tuvieron lugar en el continente americano (31\% en África, 28\% en Asia) mientras que Europa (5\%) y Oceanía (0.3\%) presentaron las tasas más bajas de homicidio a nivel regional (UNODC 2014: 40-41). Sin embargo América Latina no sólo continúa siendo la región más violenta sino que es la única región del mundo donde los homicidios se incrementaron en un 12\%. América Latina sufre más de 100.000 homicidios registrados por año. Si la tasa promedio global de homicidios es de 6.2 por cada 100000 habitantes América Central muestra promedios cuatro veces mayores (por arriba de 24 víctimas por cada 100000 habitantes). Los homicidios cometidos por la delincuencia organizada y las pandillas son muy elevados en zonas de América Central (UNODC 2014:42). $\mathrm{Y}$ aunque en algunos países como Brasil la tendencia se ha estabilizado en 
11 países, la tasa es mayor a los 10 homicidios por cada 100,000 habitantes, nivel considerado como de epidemia para la OMS (UNODC 2014:13). De la mayoría de países de América Latina analizados en el Informe Regional de Desarrollo Humano 2013-2014 del Programa de las Naciones Unidas Unidas para el Desarrollo (PNUD), en más de la mitad la tasa de homicidios aumentó, incluso en aquellos países con menores niveles de pobreza (PNUD, 2014: 15).

A pesar de las tendencias compartidas en América Latina en términos de homicidios, existen diferencias importantes entre los países de la región. La evolución del homicidio permite dividir a los países de la región en dos grupos con niveles altos, y bajos. En el primer grupo están Brasil, Colombia, Ecuador, El Salvador, Guatemala, Honduras, México, Panamá, Paraguay, República Dominicana y Venezuela, con tasas medias superiores a los 10 homicidios por cada 100,000 habitantes. En el segundo grupo, con menor tasa de homicidio, están Argentina, Bolivia, Chile, Costa Rica, Nicaragua, Perú y Uruguay (UNODC 2014:22-24). Esta heterogeneidad se observa también dentro de los países. Hay áreas de un país con una tasa de homicidio que supera hasta 10 veces las de otras ciudades o regiones. Por ejemplo en México, el estado de Chihuahua tiene una tasa de 194 homicidios por cada 100,000 habitantes, mientras que Yucatán, que es el estado más seguro, tiene una tasa de 1.74 (PNUD 2014:48). El gobierno mexicano estima que el 80\% de los homicidios ocurren sólo en 162 municipios de los 2,240 del país (IDMC 2013).

\section{Una violencia concentrada en las zonas urbanas y centrada en los jóvenes.}

El Consejo Noruego de Refugiados estima que 42 de las 50 ciudades más peligrosas del mundo se encuentran en América Latina (NRC 2014). La concentración del homicidio varía de ciudad en ciudad, entre los barrios ricos y los pobres y también entre barrios y calles. La violencia letal tiende a concentrase en las áreas urbanas más densamente pobladas, y entre las familias con bajos ingresos sea en Colombia, El Salvador o Brasil (Muggha 2012: 30). En el tiempo y el espacio, las áreas urbanas de bajos ingresos tienden a experimentar mayores tasas de violencia y victimización y dentro de estas áreas, los grupos sociales más pobres, marginales y vulnerables están en mayor riesgo que otros. Una serie de estudios de casos específicos en las grandes ciudades de Brasil confirman la concentración socio-espacial de la violencia urbana entre los grupos de ingresos más bajos. En Río de Janeiro los índices de homicidio en las favelas eran de 84 por 100,000 habitantes, mientras en barrios más prósperos alcanzaban apenas un 4 por 100,000 (Leeds 2006). Estos patrones se mantienen constantes en el tiempo. Encuestas de hogares con varias generaciones en Río de Janeiro y Sao Paolo 
revelan cómo las sucesivas generaciones de residentes de bajos ingresos en las favelas tienen un número desproporcionado de miembros de la familia que murieron en disputas entre grupos armados (Campbell 2008). Por otro lado un estudio para Belo Horizonte, Brasil, muestra que únicamente en 6 de los 81 conglomerados con favelas se concentra la violencia (Beato 2012). Esto quiere decir que no necesariamente en todas las áreas urbanas de bajos ingresos se dan niveles elevados de violencia.

El homicidio se concentra de forma alarmante entre la población joven, en particular en los varones que habitan en grandes áreas urbanas (PNUD 2014:52; UNODC, 2014). La tasa de homicidio para las víctimas masculinas de 15 a 29 años en América del Sur y Central supera en más de cuatro veces la tasa promedio global para dicho grupo de edad. Según un estudio elaborado en 2011 con base en el World Mortality Databases de la OMS, El Salvador (92.3), Colombia (73.4), Venezuela (64.2), Guatemala (55.4) y Brasil (51.6) tienen las cinco tasas de homicidio juvenil más altas del mundo (UNDP 2014: 53). Más aún, 17 países de la región figuran entre los 27 países (de un total de 83 estudiados) con más homicidio de este grupo de edad. De acuerdo con el proyecto Global Burden of Injuries, alrededor del 35\% de los homicidios en América Latina entre 1996 y 2009 tuvieron como víctimas a personas entre 10 y 25 años de edad, la mayoría de ellos hombres (UNDP 2014:53). Los hombres son las principales víctimas de los homicidios en la región, y superan las tasas de las mujeres asesinadas por cerca de 10 veces en todos los países. A pesar de ello los homicidios contra las mujeres por razones de género también han aumentado.

Aunque los datos son todavía insuficientes hay evidencia de que la tasa de homicidio de mujeres ha crecido en países como la República Dominicana y Brasil. Datos del Mapa de la Violencia 2014 - Los Jóvenes de Brasil del Instituto Sangari de Brasil ponen de manifiesto que los homicidios intencionales de mujeres son los que más que se duplicaron en el país en las últimas décadas, pasando de una tasa de 2,3 por cada 100 mil habitantes en 1980 a 4,8 homicidios por 100 mil en 2012. Esto significa un crecimiento de 111\% (Waisselfisz 2014:67). Entre las mujeres jóvenes, los datos son aún más alarmantes: la tasa de homicidios es de 7,7 por 100 mil habitantes en 2012, lo que quiere decir que cada cuatro horas una mujer menor de 30 años fue asesinada en Brasil. Pero no hay suficientes datos Las mujeres pueden ser víctimas de violencia letal no sólo por homicidios, sino también por feminicidio, definido como el asesinato de mujeres por parte de hombres por el hecho de ser mujeres. Este se presenta tanto en el ámbito público como en el privado como consecuencia de una profunda negligencia institucional de altos niveles de impunidad, que perpetúa el 
delito y aumenta la vulnerabilidad de las mujeres (PNUD 2014:54). El nuevo informe de la OMS sobre salud de la mujer y violencia doméstica contra la mujer confirma que la violencia ejercida contra la mujer constituye un problema social fundamental. Esta es tanto la consecuencia como la causa de la desigualdad de género (WHO 2014).

\section{El impacto de la violencia.}

Aunque el número de homicidios es el indicador más sistematizado de la violencia, el panorama de la violencia en la región es más amplio e incluye el robo, las amenazas de la delincuencia organizada y de delitos como la extorsión y los secuestros, la violencia de género, el delito callejero, la corrupción y la violencia ilegal por parte de actores estatales (PNUD 2014). Los robos, la extorsión y los secuestros se han casi triplicado en los últimos 25 años. En concreto el robo con violencia se ha convertido en un problema de inseguridad común para la mayoría de los países de la región. A excepción de Costa Rica, Guatemala, El Salvador y Venezuela se registra un crecimiento en la tasa de robos en todos los países. Las tasas de victimización por robo (los porcentajes de la población que dicen haber sufrido un robo en cada país) se desvían considerablemente de los registros administrativos. Una de las razones que explican esta disparidad es la falta de confianza en la capacidad de respuesta de las instituciones en distintos países, lo que puede incidir en los bajos niveles de denuncia (PNUD 2014: 57-59).

La violencia ha creado una percepción de inseguridad creciente en la ciudadanía. En todos los países de la región, incluso en los que las tasas de homicidio son relativamente bajas, el miedo a ser víctima de la violencia y el delito, incluyendo robos, se ha convertido en un sentimiento cotidiano. Cinco de cada diez latinoamericanos perciben que la seguridad en su país se ha deteriorado: hasta un $65 \%$ han dejado de salir de noche por la inseguridad y $13 \%$ reportó haber sentido la necesidad de cambiar su residencia por temor a ser víctima del delito (LAPOP-PNUD 2012). Este 13\% equivaldría a 74.8 millones de personas aproximadamente, es decir, todos los habitantes de Argentina, más los de Perú y Uruguay (PNUD 2014). Un estudio realizado en Centroamérica muestra como el $70 \%$ de los ciudadanos consideran que el crimen es una amenaza al bienestar de la nación. Un 40\% de los ciudadanos de Honduras y Guatemala tienen temor a ser víctimas de un crimen en su barrio. La relación entre la percepción de la actividad de las pandillas y el miedo de convertirse en víctima de un delito es muy estrecha. Pérez ha demostrado el impacto sicológico de la violencia de pandillas en la sensación de seguridad de los ciudadanos en barrios afectados en Honduras, Guatemala y El Salvador 
(Pérez, 2013). Con todo los costos del crimen y los niveles de victimización según muestra un estudio realizado por el PNUD y el BID en cinco países (Chile, Costa Rica, Honduras, Paraguay y Uruguay) no afectan de forma homogénea a los distintos estratos sociales ni grupos de edad (PNUD, 2014).

El temor a la violencia también tiene consecuencias en la cohesión social. Por un lado los espacios públicos vacíos o violentos dificultan su apropiación por parte de la comunidad. Por otro la inseguridad genera una preferencia por los centros comerciales cerrados en lugar del comercio tradicional y por urbanizaciones y condominios cerrados en lugar de las áreas con uso de suelo mixto y con diversidad social. Esto produce dinámicas de segregación urbana que disminuyen el espacio público y que a la larga también incide en nuevas prácticas de desarrollo inmobiliario y de producción de ciudad. La proliferación de urbanizaciones cerradas o rodeadas de muros en algunos países de América Latina responde a la percepción que tienen los miembros de estas comunidades de ser víctimas de la violencia (PNUD, 2014; Muggha 2012). Ello afecta de forma negativa la convivencia social.

Una de las consecuencias de la percepción de inseguridad, real o no, es el desplazamiento voluntario y forzado en las ciudades y hacia y desde las ciudades. El temor a la violencia hace que la gente cambie de domicilio, sus rutinas y su estilo de vida. Tanto en los barrios de ingresos más bajos como en los de ingresos más altos son los hogares más acomodadas de los mismos y en particular los que tienen niños en edad escolar y pre-escolar los que tienden a irse primero, quedándose a menudo los más pobres. Este éxodo de capital humano produce en muchas ocasiones, en barrios desprovistos de sus pobladores mejor educados y más preparados, un declive en la calidad y cantidad de los servicios, un descenso en redes de mercado que conlleva una pérdida del valor de la propiedad, una paralización de las inversiones y, en definitiva, una agudización de la pobreza (Muggha, 2012). A este éxodo de las personas más acomodadas se une el de las personas que pertenecen a grupos vulnerables que históricamente han estado excluidas de los beneficios de los modelos de acumulación, excluidas de la participación política y culturalmente invisibilizadas (Nubia Bello, 2004). Este desplazamiento por sus consecuencias humanitarias merece una consideración aparte.

\section{EI desplazamiento forzado de personas por causa de la violencia criminal}

A finales de 2013, por lo menos 6,3 millones de personas habían sido objeto de desplazamiento forzado interno por causa de la violencia en las 
Américas $^{5}$. Las personas desplazadas sufren vulnerabilidades y necesidades específicas. No sólo pierden sus hogares, lo que los obliga a buscar alojamiento alternativo; pierden además sus medios de vida y de supervivencia y sus redes sociales y culturales, lo que suele redundar en empobrecimiento, marginación, exclusión de los servicios básicos e incluso estigmatización. En Colombia el conflicto prolongado en el país es la principal causa del desplazamiento, pero también lo es la expansión de la violencia criminal que ha forzado a decenas de miles de personas a abandonar sus hogares en toda la región, incluyendo países como México, Honduras, Guatemala y El Salvador. También en Panamá está aumentando la violencia relacionada con las drogas en los últimos años como consecuencia de la presencia de los grupos criminales colombianos descendientes de los paramilitares, las denominadas BACRIM, que están utilizando cada vez más Panamá como ruta de tráfico, corrompiendo a funcionarios e influenciando a las bandas locales (Yagoub 2014).

El control territorial por parte del crimen organizado, además de las disputas y luchas internas entre diferentes grupos criminales, tales como las alianzas locales con los grandes cárteles, provocan el desplazamiento forzado de las víctimas, que huyen inicialmente de las comunidades de origen. A través de acciones como las extorsiones, pago de cuotas periódicas (cuota o "impuesto de guerra"), amenazas a la vida, violaciones sexuales, asesinatos, tortura, reclutamiento forzado de jóvenes, niños y niñas, y secuestros provocan un impacto en los individuos y sus familias. Las amenazas se concretan no sólo en estas comunidades; también se han reportado en las zonas de tránsito migratorio asesinatos masivos de migrantes indocumentados, extorsiones, robos, violaciones sexuales y el reclutamiento forzado de jóvenes para llevar a cabo asesinatos masivos (masacres) en otros países (CIDEHUM 2014: 1116). El desplazamiento forzado por la violencia criminal es especialmente preocupante por su falta de reconocimiento por las autoridades. La ausencia de datos confiables y verificables impide la comprensión de las verdaderas dimensiones del fenómeno. La mayoría de las informaciones disponibles proceden de observadores de la sociedad civil. Por ejemplo, el desplazamiento en El Salvador no aparece registrado por el principal centro mundial de análisis del desplazamiento forzado interno el IDMC. Sin embargo es el único país que cuenta con una encuesta nacional sobre desplazamiento relativamente sistemática (Cantor 2014:4).

La gran mayoría de desplazados se encuentra en Colombia, donde la cifra

\footnotetext{
${ }^{5}$ Se consideran así las personas o grupos de personas que se han visto forzadas u obligadas a escapar o huir de su hogar o de su lugar de residencia habitual, en particular como resultado o para evitar los efectos de un conflicto armado, de situaciones de violencia generalizada, de violaciones de los derechos humanos o de catástrofes naturales o provocadas por el ser humano, y que no han cruzado una frontera estatal internacionalmente reconocida. Véase (Naciones Unidas-Comisión de Derechos Humanos, 1998).
} 
ha aumentado de forma constante durante un período de diez años y alcanza ahora los 5,7 millones. Según el gobierno colombiano, más de 150.000 personas fueron desplazadas en 2012, mientras que CODHES, una respetada organización no gubernamental (ONG) colombiana, calcula en casi 260.000 el número de desplazados ese año ${ }^{6}$. Los niveles de desplazamiento son particularmente elevados en los departamentos del Pacífico, en Antioquia, Putumayo y Norte de Santander. El desplazamiento produce el desarraigo de los pobladores rurales y su asentamiento en las condiciones y zonas más adversas de las ciudades intermedias y las grandes capitales de todo el país como Bogotá, Medellín, Pereira, Armenia, Cali, Quibdó, entre otras. Aunque la población desplazada es muy heterogénea y cada vez tiende a diversificarse, los desplazados son en su gran mayoría campesinos pobres y personas pertenecientes a comunidades étnicas, afrocolombianas e indígenas. A la discriminación histórica que sufren se suma la ausencia de una cultura moderna de solidaridad y la ausencia de una política especial capaz de reconocer sus particulares necesidades y derechos (Ceballos Bedoya, 2013). Como señala un estudio de CODHES en las ciudades de Buenaventura, Tumaco y Soacha en Colombia durante muchos años la actitud típica de las autoridades locales ha sido incluso la de negar la atención a las personas desplazadas (CODHES, 2013). Del mismo modo, la actitud de muchos pobladores urbanos es la de considerar a las víctimas del desplazamiento como apátridas, como competidores por servicios y no como los nuevos habitantes de la ciudad, como ciudadanos de un mismo país y de un mismo Estado que ha fracasado en su tarea de protección.

El desplazamiento intraurbano (DFI) en particular evidencia la emergencia de poderes coactivos de tipo fáctico que buscan imponer su dominio en zonas urbanas en las que el Estado no ofrece las garantías plenas de ciudadanía. En Colombia en particular el DFI es la muestra de la extensión del conflicto armado a las ciudades. El DFI radicaliza la demanda por empleos, vivienda e ingresos en ciudades segregadas, que tampoco han sido objeto de reformas urbanas basadas en el principio de inclusión. De allí el predominio del asistencialismo, la informalidad y la marginalidad como formas de vida urbana de la mayor parte de las víctimas del desplazamiento y de enormes contingentes de pobres en contextos urbanos. En estas se mezclan los conflictos sociales urbanos con las diferentes dinámicas que alientan el conflicto: los movimientos de delincuencia característicos de las urbes y la existencia de estructuras criminales con presencia nacional alimentadas por un accionar basado en el paramilitarismo (las bandas criminales conocidas como BACRIM (CODHES, 2013). Sólo en la ciudad de Buenaventura en la costa del Pacífico,

\footnotetext{
${ }^{6}$ Conforme indican datos revisados recientemente por el gobierno, más de 5 millones de colombianos han sido desplazados internamente desde 1985. Según CODHES esta cifra asciende a 5,701.996 desde 1985. En promedio anualmente 211.178 personas se han desplazado dentro del país 
que alberga a una importante población afrocolombiana, los grupos sucesores de paramilitares han provocado en 2013, como denuncia la organización Human Rights Watch, el desplazamiento forzado de más de 13,000 personas, además de las desapariciones probablemente de cientos de personas que son descuartizadas y arrojadas a la bahía o a los manglares, o enterrados en fosas clandestinas (HRW, 2014:4).

Como señalábamos en la introducción, durante los últimos años el nivel y la intensidad de la violencia en el Triángulo Norte de Centroamérica y México ha aumentado por la rápida expansión de grupos criminales en la región. Desde 2007, México se ha enfrentado a una ola sin precedentes de violencia criminal, con la lucha de las organizaciones criminales entre sí y, en menor medida, contra el Estado. La estrategia que México ha seguido para combatir el crimen organizado en los últimos años no sólo destaca el aumento de la violencia, sino también el alto número de desplazados que los enfrentamientos armados provocan (IDMC, 2014). La intervención de las fuerzas armadas en las rutas de los grupos rivales ha provocado una guerra sobre el terreno previamente acordado entre los cárteles para las rutas del tráfico de droga. Se estima que desde 2007 a 2010, el número de desplazados fue de 230,000, de los que al menos 115,000 cruzaron la frontera; el resto se ha movido en México, en los estados de Chihuahua, Durango, Coahuila y Veracruz, según el Centro de Monitoreo de Desplazamiento Interno (IDMC, 2011). Cantor, basándose en datos preliminares de algunos investigadores, señala que un 2 por ciento de la población que vive en el país, 1,65 millones de personas, ha cambiado de residencia en los cinco años comprendidos entre 2006 y 2011 , debido a la amenaza o riesgo de violencia lo que sugiere un promedio de media de 330.000 desplazados internos por año (Cantor 2014:3). Sólo en 2013 se cifran en alrededor de 20,000 los desplazados internos. Aunque no hay cifras globales del número de personas desplazadas disponibles, la correlación entre los datos del censo y los datos sobre homicidios y crímenes violentos muestra que la violencia criminal causó el desplazamiento de los estados más afectados por la violencia de los cárteles de drogas en 2013, a saber, Baja California, Chihuahua, Coahuila, Durango, Guerrero, Michoacán, Nuevo León, San Luis Potosí, Sinaloa, Sonora, Tamaulipas y Veracruz (IDMC, 2013).

A falta de registros oficiales sobre la cantidad de personas desplazadas forzadas por el crimen organizado en la región centroamericana, una aproximación a la magnitud de esta situación puede darse, como ha hecho el Centro Internacional para los Derechos Humanos de los Migrantes (CIDEHUM), revisando los índices de homicidios en los países concernidos, la identificación de zonas de riesgo y las zonas en las que se ha reportado el desplazamiento forzado por parte del crimen organizado, las cifras de las 
personas deportadas y expulsadas desde países de México y Estados Unidos ${ }^{7}$ y las solicitantes de la condición de refugiados por persecución del crimen organizado (CIDEHUM 2012:5). Un estudio realizado por el CIDEHUM a solicitud del Alto Comisionado de las Naciones Unidas para los Refugiados, ACNUR, observa como esas cifras han ido en aumento durante los últimos años. El aumento se corresponde con el fortalecimiento de una estructura funcional y organizada muy importante a nivel territorial y social, que ha atravesado estos países desde una perspectiva (pasadizo de drogas, armas, traficantes de migrantes y tratantes de personas), que afecta la dinámica de movilidad humana, directamente vinculada a la violencia y la falta de seguridad y protección (CIDEHUM 2012:11).

El crimen organizado se concentra en áreas estratégicas, principalmente en zonas fronterizas y centros urbanos de las principales ciudades de la región centroamericana (CIDEHUM 2012: 11). El patrón que se repite en El Salvador, Guatemala y Honduras es el siguiente: primero, en las zonas de origen del desplazamiento apropiadas por el crimen organizado como "narcoterritorios", la población se encuentra abocada a ser reclutada por este o a salir de los territorios; segundo, al desplazarse hacia el interior del país en búsqueda de otras zonas o territorios, las personas forzadas a desplazarse son identificadas por los agentes criminales, especialmente aquellas testigos de crímenes y quienes hayan denunciado en las instancias públicas correspondientes; y, tercero, ante la situación de desprotección estas personas se ven obligadas a un segundo desplazamiento esta vez externo, principalmente hacia Norteamérica. Desde 2007, a raíz de la represión del gobierno mexicano contra los cárteles de la droga, los cárteles han aumentado sus operaciones y los niveles de violencia en Guatemala (IDMC 2014). En Honduras la violencia criminal por parte de bandas locales y las organizaciones transnacionales de narcotráfico han obligado al menos a 17.000 personas a huir de sus hogares en Honduras en los últimos años, según señala el IDMC citando observadores de la sociedad civil (IDMC 2014). A pesar de que su extensión territorial es parecida a Guatemala, este país, según afirma el CIDEHUM "ofrece muy pocas opciones de desplazamiento forzado interno, ya que el crimen organizado se ha apropiado de gran cantidad de territorios en el país" (CIDEHUM 201:24).

\section{Conclusiones y reflexiones finales.}

El objetivo de este artículo ha sido poner en evidencia el desafío que el fenómeno de la violencia representa a la seguridad humana en América Latina.

\footnotetext{
${ }^{7}$ Los resultados este estudio señalan de que existe una tendencia de parte del crimen organizado a captar y/o explotar a las personas centroamericanas deportadas y expulsadas de México y Estados Unidos de América a sus países de origen.
} 
Se ha mostrado un panorama de violencia más allá de las tasas conocidas de homicidios, señalando el impacto de la violencia en la vida de las personas, en particular una dimensión menos conocida y muy poco estudiada todavía como es el desplazamiento forzado de población a causa del crimen organizado.

La región sufre una violencia endémica que no tiene su campo privilegiado de acción en las zonas rurales, sino en las urbanas y, sobre todo, en las zonas pobres, segregadas y excluidas de las grandes ciudades, donde la presencia del Estado es o prácticamente inexistente o insuficiente e inoperante. Una violencia considerada crónica porque se reproduce afectando las relaciones sociales y la práctica ciudadana en la región. Además de las consecuencias directas que tiene la violencia en la vida y los medios de vida de las personas, esta produce un sentimiento de temor y vulnerabilidad y repercute de forma negativa en el espacio público y el tejido social. Asimismo restringe la movilidad o fuerza el desplazamiento de las personas y limita el acceso al trabajo y a la educación en un círculo vicioso en que la violencia, la pobreza y la desigualdad se refuerzan mutuamente empeorando la situación socioeconómica de las personas ya pobres. La incapacidad de los gobiernos locales para regular y monopolizar el uso de la violencia legítima en sus territorios está rompiendo el contrato social que vincula a los gobernantes y a los ciudadanos. El problema del desplazamiento forzado evidencia la falta de seguridad y desprotección de las personas y la existencia de territorios estratégicos controlados por el crimen organizado.

El informe regional del PNUD Seguridad Ciudadana con Rostro Humano: Diagnóstico y propuestas para América Latina subraya que "si bien algunas amenazas - como la delincuencia organizada, y especialmente el narcotráficosuelen ser señaladas de manera recurrente para explicar por qué América Latina es una de las regiones más inseguras del mundo, lo cierto es que las dinámicas regionales, nacionales y locales de la inseguridad son mucho más diversas. Incluso, muchas de estas amenazas, como la corrupción, la violencia de género (en particular la violencia intrafamiliar) y el delito callejero no están fincadas necesariamente en redes criminales transnacionales, sino que se originan y se manifiestan prácticamente en el ámbito de lo local" (PNUD 2014: 7). En otras palabras, que en el conjunto de las amenazas a la seguridad humana en su interacción y su retroalimentación en el ámbito local subyace la explicación de los crecientes índices de crimen y violencia que afectan cotidianamente a los ciudadanos de la región. Y que aunque se manifiesten en distintos espacios de interacción social, desde el hogar a la comunidad hasta el espacio público, estas amenazas están relacionadas y pueden retroalimentarse y generar mayores ciclos de violencia.

Ahora bien, aun sin desestimar el argumento del PNUD, no se puede soslayar la importancia de la relación entre la delincuencia organizada y el narcotráfico 
con la violencia en la región. Un reciente informe de la Organización de Estados Americanos apunta que sin lugar a duda un número significativo de casos de homicidios dolosos en AL está asociado "a la actividad del crimen organizado relacionada a su vez con la economía ilegal y específicamente con el tránsito de drogas desde países principal, aunque no exclusivamente, productores hacia países principal, aunque no exclusivamente, consumidores" (OEA, 2013:7980). Si ampliando el panorama se incluye el desplazamiento forzado interno y externo a causa del crimen organizado como una situación real y emergente está claro que la violencia en la región es un desafío que requiere de respuestas desde una perspectiva humanitaria y de seguridad humana más allá de políticas de seguridad nacional y regional y control migratorio. Hasta que los Estados y las organizaciones internacionales no reconozcan el problema de la violencia en toda en su dimensión se brinda más oportunidades al crimen organizado para continuar impune y seguir generando desprotección y falta de seguridad humana 


\section{Referencias bibliográficas:}

Aróstegui Sánchez, Julio, "Violencia, sociedad y política: la definición de la violencia”, Ayer, 13, 1994, págs. 17-56.

Beato, Claudio, Crimes y Ciudades, Belo Horizonte, UFMG, 2012.

Briceño-León, Roberto, "La violencia homicida en América Latina", América Latina Hoy, n 50, 2008, pp. 103-116.

Caldeira, Teresa P.R., City of Walls: Crime, Segregation and Citizenship in Sao Paulo. Berkeley, University of California, 2000.

Campbell, Tim., Which way out? Favela as Lethal Hall of Mirrors, Whashington D.C.., Woodrow Center for Scholars and Fetzer Institute, 2008.

Cantor, David James, The New Wave: Forced Displacement Caused by Organized Crime in Central America And Mexico, Refugee Survey Quarterly, 2014 pp. 1-35, Downloaded from http://rsq.oxfordjournals.org/ by guest on June 13, 2014.

Ceballos Bedoya, María Adelaida, "El desplazamiento forzado en Colombia y su ardua reparación”, Araucaria. Revista Iberoamericana de Filosofía Política y Humanidades, año 15, n²9, 2013, pp.169-188.

CEPAL, Perspectivas económicas de América Latina 2014: logística y competitividad para el desarrollo, OCDE/CEPAL/CAF, 2013.

Churruca, Cristina , Human Security as a policy framework: Critics and Challenges [en Anuario de Accion Humanitaria y Derechos Humanos/Yearbook on Humanitarian Action and Human Rights 2007, Bilbao,Universidad de Deusto, 2007], págs.15-35.

CIDEHUM, Diagnóstico "Desplazamiento Forzado y Necesidades de Protección, generados por nuevas formas de Violencia y Criminalidad en Centroamérica", CIDEHUM-ACNUR, 2012.

CODHES, Comisión de Seguimiento a la política pública sobre desplazamiento forzado (2009): El reto ante la tragedia humanitaria del desplazamiento forzado: Reparar de manera integral el despojo de tierras y bienes, Colombia, Comisión de seguimiento a la política pública sobre desplazamiento forzado y Consultoría para los Derechos Humanos, CODHES accesible en: http://www.internal-displacement. org/8025708F004CE90B/(httpDocuments)/9C9025369DCE9579C12575 E0005439CA/\$file/Vol_5_TIERRAS.pdf

CODHES, Desplazamiento intraurbano y soluciones duraderas. Una aproximación a los casos de Buenaventura, Tumaco y Soacha, Bogotá, CODHES-ACNUR, 2013.

ECHO, Humanitarian Implementation Plan (HIP) Central America / Mexico, ECHO/-CM/BUD/2013/91000, 2013 , en http://ec.europa.eu/echo/.../ HIPs/central_america en.pdf. 
HASOW (Humanitarian Action in Other Situations other tan War) FIRST HASOW INTERNATIONAL CONFERENCE PROCEEDINGS, The Politics of Protection and the Future of Humanitarian Practices, marzo de 2013 en http://www.hasow.org/Job/Interna/107

HASOW (Humanitarian Action in Situations Other than War) FIRST HASOW INTERNATIONAL CONFERENCE The Politics of Protection and the Future of Humanitarian Practices en marzo de 2013 en http://www.hasow. org/Job/Interna/107 p.9.

Human Rights Watch, http://www.hrw.org/es/world-report/2014/countrychapters/121995

Human Rights Watch, La crisis en Buenaventura Desapariciones, desmembramientos y desplazamiento en el principal puerto de Colombia en el Pacifico, Estados Unidos de América, Human Rights Watch, 2014.

IDMC , Guatemala: Internal displacement in brief, As of December 2011, http://www.internal-displacement.org/americas/guatemala/summary accedido el 17 de junio 2013

IDMC, Honduras: Internal displacement in brief, As of December 2013, http:// www.internal-displacement.org/americas/honduras/summary accedido el 17 de junio 2013.

IDMC, Internal Displacement: Global Overview of Trends and Developments in 2010, March 2011.

Internal Displacement Monitoring Centre (IDMC)-Norwegian Refugee Council (NRC), Briefing for the Human Rights Council's Universal Periodic Review 17th session of the UPR Working Group (21 October - 1 November 2013) Mexico 22 October 2013.

Jutersonke, O., Muggah, R. and Rodgers, D. (2009). "Gangs, Urban Violence and Security Interventions in Central America", Security Dialogue 40 (4-5). $\quad$ http://eprints.lse.ac.uk/28428/1/Gangs, urban_violence and security interventions (LSERO version).doc.pdf

Kevin Sabage and Robert Muggah, "Urban Violence and Humanitarian Action: Engaging the Fragile City", The Journal of Humanitarian Assistance, January 19 2012, en http://sites.tufts.edu/jha/archives/1524.

Latin American Public Opinion Project (LAPOP, 2012), Latin American Public Opinion Project 2012, Vanderbilt University. Disponible en: http://www. vanderbilt.edu/lapop/

Leeds, Elizabeth, Rio de Janeiro [en Koonings, Kees. y Krujit, Dirk, eds.: Fractured Cities: Social Exclusion, Urban Violence \& Contested Spaces in Latin America, New York, Zed Books, 2006]

Marilena Adams, Tani, La violencia crónica y su reproducción: Tendencias en las relaciones sociales y la democracia en América Latina, Washington, 
Woodrow Wilson Wilson Center for International Scholars-Instituto Internacional de Aprendizaje para la Reconcialiación Social, 2013.

Muggha, Robert, Researching the Urban Dilemma: Urbanization, Poverty and Violence, International Development Research Centre (IDRC) and the United Kingdom's Department for International Development (DFID), 2012 accesible en http:/www.idrc.ca/EN/Programs/Social and_Economic_Policy/Governance_Security_and_Justice/Pages/ ArticleDetails.aspx?PublicationID $=109 \overline{6}$.

Norwegian Regugee Council, Key Findings 2013: AMERICAS http://www.nrc.

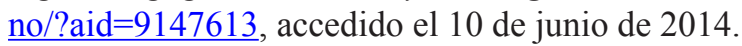

OMS, Informe mundial sobre la violencia y la salud .Resumen Ejecutivo, Washington, Organización Panamericana de la Salud para la Organización Mundial de la Salud, 2002.

Pearce, Jenny, Violence, Power and Partcipation: Building Citizenship in Contexts of Chronic Violence, IDS Working Paper 274, Brighton, Institute of Development Studies, 2007.

Pearce, Jenny; McGee, Rosemary and Wheeler, Joanna, Violence, Security and Democracy: Perverse Interfaces and their Implications for States in the Global South, IDS Working Paper 357, Brighton, Institute of Development Studies, 2011.

Pérez, Orlando J., "Gang Violence and Insecurity in Contemporary Central America", Bulletin of Latin American Research, Journal of the Society for Latin American Studies, 32-1 (2013), págs. 217-234.

PNUD, Informe sobre desarrollo humano para América Central 2009-2010. Abrir espacios para la seguridad ciudadana y el desarrollo humano, Nueva york, PNUD, 2010.

PNUD, Informe sobe el desarrollo humano del Caribe 2012: Desarrollo Humano y transición hacia una mejor seguridad ciudadana, Nueva york, PNUD, 2013.

PNUD, Informe Regional de Desarrollo Humano 2013-2014. Seguridad Ciudadana con Rostro Humano: Diagnóstico y propuestas para América Latina, Nueva york, PNUD, 2014.

Savage, Kevin and Muggha, Robert, "Urban Violence and Humanitarian Action: Engaging in the Fragile City", The Journal of Humanitarian Assistance, 19 (2012) en http://sites.tufts.edu/jha/archives/1524.

UNODC, Global Study on Homicide 2013 del Oficina de Naciones Unidas sobre Drogas y Crimen, Geneve, UNODC, 2014.

Waisselfisz, Julio Jacobo, Mapa da violência 2013. Os jovens do Brasil, Brasília, Instituto Sangari-Ministério da Justiça, 2014.

WHO, Global and regional estimates of violence against women: prevalence and health effects of 
Yagoub, Mimi, Panamá advierte sobre incursión de BACRIM colombiana: Informe, InSightCrime, 09 Julio 2014, http://es.insightcrime.org/noticiasdel-dia/panama-advierte-sobre-incursion-bacrim-colombiana-informe, accedido 10 de julio de 2014 . 
